\title{
TRUNCATION ERRORS IN CYLINDRICAL NEAR TO FAR FIELD TRANSFORM. A PLANE WAVE SYNTHESIS APPROACH
}

J. Romeu, L. Jofre.

\section{ABSTRACT}

Cylindrical Near to far field antenna transformation is expressed as a plane wave synthesis problem where a plane wave is produced by a cylindrical current distribution that encloses the Antenna Under Test (AUT). The current distribution that produces the plane wave is directly derived from the near to far field transformation algorithm.

\section{INTRODUCTION}

In near to far field antenna measurements the Antenna Under Test (AUT) near fields are measured on an enclosing surface close to the AUT. The AUT far fields are found after processing these near fields. Planar, cylindrical and spherical scanning surfaces are used in practice. In order to perform the near to far field transformation, the near fields must be known on the entire surface that encloses the AUT. In the planar and cylindrical case, this condition implies that the surface must spread to infinity. In practice an error is associated to the limited extent of the measurement surface. This error is usually known as truncation error. The effect of this error on the antenna far field pattern is among other factors heavily dependent on the AUT characteristics. The formulation of the near to far field transformation as a plane wave synthesis process allows to express the truncation error as a deterioration of the synthesized wave. In this way the performance of the near field range can be expressed independently of the AUT. On the other hand, plane wave quality is a common figure of merit in compact or far field ranges.

\section{EXPRESSION OF THE CYLINDRICAL NEAR TO FAR FIELD TRANSFORMATION AS A PLANE WAVE SYNTHESIS}

All antenna pattern measurement schemes find the AUT response to an incident plane wave. In near field measurements the AUT response to a plane wave is somehow obtained, although it does not appear explicitly in the transformation algorithm.

In cylindrical antenna near field measurements, a probe scans the AUT near fields on a cylindrical surface (fig. 1). Consider a reciprocal case where the probe is emitting and the AUT receiving. Let us define $v_{o c}^{(p)}\left(\rho_{0}, z^{\prime}, \phi^{\prime}\right)$ as the open voltage measured on the AUT access port when the probe is placed at $\rho_{0}, z^{\circ}, \phi^{\prime}$ and fed with an unitary voltage at its input. The superindex (p) can be 1 or 2 and reflects the fact that two probes are required to perform the near to far field transformation. Consider now that a cylindrical array of probes is constructed and each probe is fed at its input by a voltage given by $\omega\left(z^{\prime}, \phi^{\prime}\right)$. The AUT response to the cylindrical array can be found as: 


$$
v^{(p)}=\int_{0}^{2 \pi} \int_{-\infty}^{\infty} \omega\left(z^{\prime}, \phi^{\prime}\right) v_{o c}^{(p)}\left(z^{\prime}, \phi^{\prime}\right) d \phi^{\prime} d z^{\circ}
$$

Consider now that $\omega\left(z^{\prime}, \phi^{\prime} ; k_{z 0}, \phi_{0}\right)$ expresses the weight that has to be given to the each element of the cylindrical array placed at point $\rho_{0}, \phi^{\prime}, z^{\circ}$, to produce a plane wave propagating in direction $k_{z 0}=k \cos \theta_{0}, \phi_{0}$ and a given polarization $\hat{e}$, where

$$
\begin{gathered}
\hat{e} \cdot \vec{k}_{0}=0 \\
\vec{k}_{0}=k \sin \theta_{0} \sin \phi_{0} \hat{x}+k \operatorname{sen} \theta_{0} \cos \phi_{0} \hat{y}+k \cos \theta_{0} \hat{z}
\end{gathered}
$$

In this case the voltage $v^{(p)}$ induced at the AUT terminals is the AUT response to a plane wave propagating in the $\vec{k}_{0}$ direction and that given polarization $\hat{e}$. For a reciprocal antenna this voltage is the same as the amplitude of the radiated field in the same direction and polarization. The general problem of finding the weights $\omega\left(z^{\prime}, \phi^{\prime} ; k_{z 0}, \phi_{0}\right)$ does not have a solution for a finite length cylinder. An iterating approach to find weight functions that produce good approximations to plane waves in a certain volume with finite sources is found in [1]. In our case we are interested in finding the weights that are inherently implicit in the transformation algorithm. The cylindrical near to far field transformation is well described elsewhere $[2,3]$, and can be written in the following way:

$\left[\begin{array}{ll}E_{0} & \left(k_{z 0}, \phi_{0}\right) \\ E_{0} & \left(k_{z 0}, \phi_{0}\right)\end{array}\right]=\frac{e^{-j k r}}{r} \int_{0}^{2 \pi} \int_{-\infty}^{\infty} \bar{\omega}\left(\phi^{\prime}, z^{\prime} ; k_{z 0}, \phi_{0}\right)\left[\begin{array}{ll}v_{o c}^{(1)} & \left(\phi^{\prime}, z^{\prime}\right) \\ v_{o c}^{(2)} & \left(\phi^{\prime}, z^{\prime}\right)\end{array}\right] d \phi^{\prime} d z^{\prime}$

with

$\bar{\omega}=\frac{\eta}{16 \pi^{3} \operatorname{sen} \theta_{0}} e^{j k_{z 0} z} \sum_{n}^{k_{0} p_{0}} j^{n+1} \frac{1}{\Delta\left(n, k_{z 0}\right)} e^{j n\left(\psi_{0}-k\right)}\left[\begin{array}{cc}-a_{n}^{(2)} & a_{n}^{(1)} \\ -j b_{n}^{(2)} & j b_{n}^{(1)}\end{array}\right]$

and

$$
\Delta\left(n, k_{z}\right)=a_{n}^{(1)}\left(-k_{z}\right) b_{n}^{(2)}\left(-k_{z}\right)-a_{n}^{(2)}\left(-k_{z}\right) b_{n}^{(1)}\left(-k_{z}\right)
$$

In these expressions $E_{\theta}\left(k_{z 0}, \phi_{0}\right)$ and $E_{\phi}\left(k_{z 0}, \phi_{0}\right)$ are the AUT far fields in direction $k_{z 0}, \phi_{0}$, and polarization $\theta$ and $\phi$ respectively. The voltages $v_{o c}^{(1)}\left(\phi^{\prime}, z^{\prime}\right)$ and $v_{o c}^{(2)}\left(\phi^{\prime}, z^{\prime}\right)$ are the measured voltages by each of the probes required to perform the transformation in position $\phi^{\prime}, z^{\prime}$. And the coefficients $a_{n}^{(1)}, b_{n}^{(1)}, a_{n}^{(2)}, b_{n}^{(2)}$ are related to the cylindrical wave expansion of the probe radiated fields.

Notice that $\bar{\omega}\left(\phi^{\prime}, z^{\prime} ; k_{z 0}, \phi_{0}\right)$ are the weights that have to be given to probes 1 and 2 , in position $\phi^{\prime}, z^{\prime}$ to produce a plane wave propagating in direction $k_{z 0}, \phi_{0}$. If we consider that probe 1 is a vertical infinitesimal dipole and probe 2 a horizontal infinitesimal dipole, the current distribution to produce a vertical plane wave is:

$\vec{J}(z, \phi)=-\frac{1}{2 \pi^{2}} e^{j k_{z 0} z} \sum_{n} j^{n+1} e^{j n\left(\phi_{0}-\phi\right)} \frac{1}{\operatorname{sen} \theta_{0} H_{n}^{(2)}\left(k_{\rho_{0}} \rho_{0}\right)} \frac{\delta\left(\rho-\rho_{0}\right)}{\rho} \hat{z}$ 
and to produce a horizontal plane wave is:

$\vec{J}(z, \phi)=-\frac{1}{2 \pi^{2}} e^{j k_{z 0} z} \sum_{n} j^{n+1} e^{j n\left(\phi_{0}-\phi\right)}\left[-j \frac{k_{z 0} n}{k_{\rho_{0}}^{2} \rho_{0} H_{n}^{(2) \cdot}\left(k_{\rho \rho_{0}} \rho_{0}\right)} \hat{z}+\right.$

$\left.+j \frac{1}{H_{n}^{(2)} \cdot\left(k_{\rho_{0}} \rho_{0}\right)} \delta\right] \frac{\delta\left(\rho-\rho_{0}\right)}{\rho}$

with $k_{z 0}^{2}+k_{\rho 0}^{2}=k^{2}$.

Each one of these current distributions produce the following fields inside of the cylinder

$$
\begin{aligned}
& \vec{E}=\frac{j \omega \mu}{4 \pi} e^{j \vec{k}_{0} \cdot \vec{r}} \hat{\theta}_{0} \\
& \hat{\theta}_{0}=\cos \theta_{0} \cos \phi_{0} \hat{x}+\cos \theta_{0} \sin \phi_{0} \hat{y}-\sin \theta_{0} \hat{z}
\end{aligned}
$$

and

$$
\begin{aligned}
& \vec{E}=\frac{j \omega \mu}{4 \pi} e^{j \vec{k}_{0} \cdot \vec{r}} \phi_{0} \\
& \hat{\phi}_{0}=-\sin \phi_{0} \hat{x}+\cos \phi_{0} \hat{y}
\end{aligned}
$$

However when the cylinder length is finite these ideal plane waves deteriorate. The resulting synthesized wave results from considering the current distributions given by equations 6 and 7, but with a finite length along the $\mathrm{z}$ axis

\section{ERROR CRITERIA}

In order to quantify the synthesized plane wave error, it is convenient to define the following error function [4]:

$$
T(\vec{r})=\frac{\left|\vec{E}_{e r r}(\vec{r})\right|^{2}+\eta^{2}\left|\vec{H}_{e r r}(\vec{r})\right|^{2}}{\left|\vec{E}_{p l}(\vec{r})\right|^{2}+\eta^{2}\left|\vec{H}_{p l}(\vec{r})\right|^{2}}
$$

where the deviation from the ideal plane wave is defined as

$$
\begin{aligned}
& \vec{E}_{e r r}=\vec{E}-\vec{E}_{p l} \\
& \vec{H}_{e r r}=\vec{H}-\vec{H}_{p l}
\end{aligned}
$$

This error function is a scalar function that considers the error in the electrical and magnetic field in magnitude and phase. It is also interesting to define the root mean square value of the plane wave error function as: 


$$
T_{r m s}=\frac{1}{S}\left[\int_{S}|T(\vec{r})|^{2} d S\right]^{\frac{1}{2}}
$$

\section{RESULTS}

By using expressions (1) (2) it is possible to compute the synthesized plane wave inside the measurement cylinder. For this purpose the current distributions have been discretized, and the field inside of the cylinder has been computed as the superposition of the radiated fields of a cylindrical array of infinitesimal dipoles. Figure 2 shows the electrical field components along the $z$ axis when a vertical plane wave is synthesized. Figure 3 shows the plane wave error function along the $z$ axis, for vertical plane wave, for different incident angles. Finally, figure 4 shows the rms value of the plane wave error computed along the $\mathrm{z}$ axis for different cylinder lengths and in function of the incident angle. The asterisk shows the validity angle $\left(\theta_{v}\right)$ for the different cylinders defined as:

$$
\tan \theta_{v}=\frac{z_{m}-z_{a}}{2\left(\rho_{0}-a\right)}
$$

Notice that for the different cylinder lengths, the rms value of the plane wave error function is approximately the same when the plane wave incident angle is the validity angle. On the other hand, in figure 4 the top horizontal line is the rms value of the plane wave error considering only a quadratic phase error along the $z$ axis. The maximum error is $\pi / 8$ as in the usual far field criteria.

\section{CONCLUSIONS}

The cylindrical near to far field transformation has been expressed as a plane wave synthesis process. In this way the truncation error due to the finite length of the measurement cylinder can be studied as a deterioration of the synthesized plane wave. By using an error criteria it is possible to compare a cylindrical near field antenna measurement facility with a compact or a far field range on plane wave quality basis.

\section{REFERENCES}

[1] Bennet. J.C, Schoessow. E.P.,' Antenna near field/far field transformation using plane wave synthesis technique', IEE Proc., vol. 125, No. 3, March 1978.

[2] Leach. W.M., Paris. D.T., 'Probe Compensated Near-Field Measurements on a Cylinder.' IEEE Trans. Antennas Propagat., vol. AP-21, July 1973.

[3] Borgiotti. G.V. 'Integral Equation Formulation for Probe Corrected Far-Field Reconstruction from Measurements on a Cylinder', vol. AP-26, July 1978.

[4] Hansen. J.E., 'Spherical Near Field Antenna Measurements', Peter Peregrinus Ltd., London 1988, Cap. 7. 


\section{ACKNOWLEDGMENT}

This work has been supported by the Spanish Committee for Scientific and Technical Research (CICYT) under grant 91-1034.

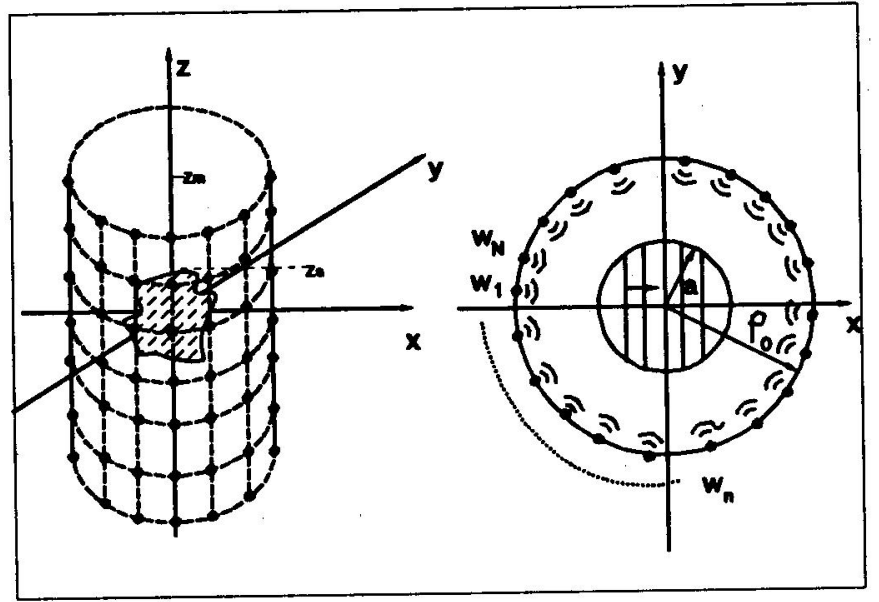

Figure 1. Synthesis geometry.

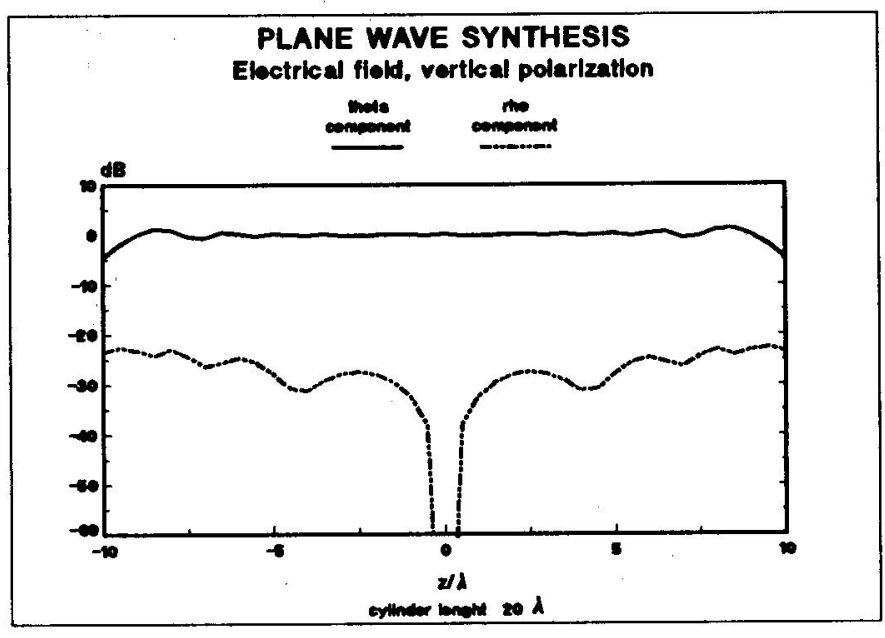

Figure 2. Synthesized electrical field.

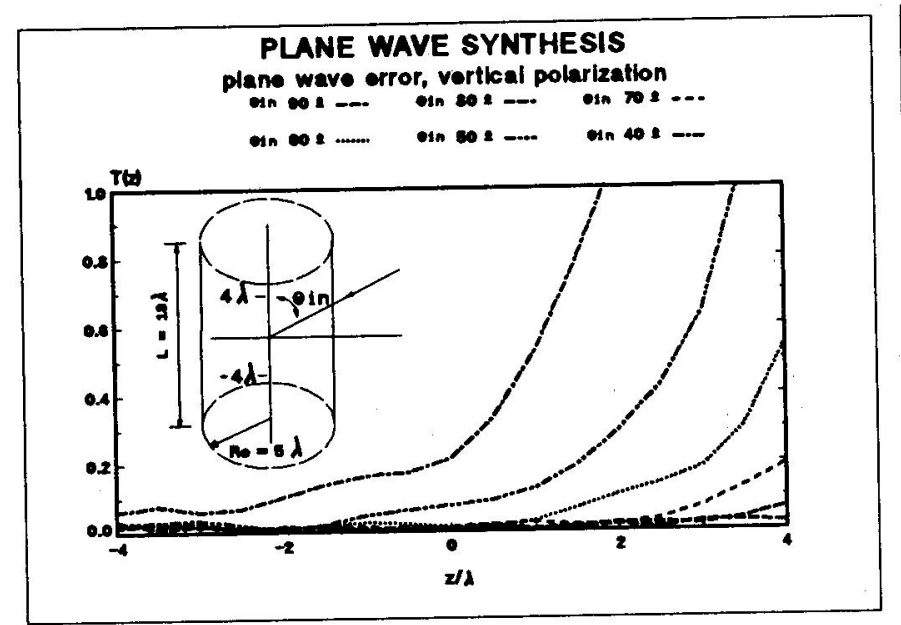

Figure 3. Plane wave error function.

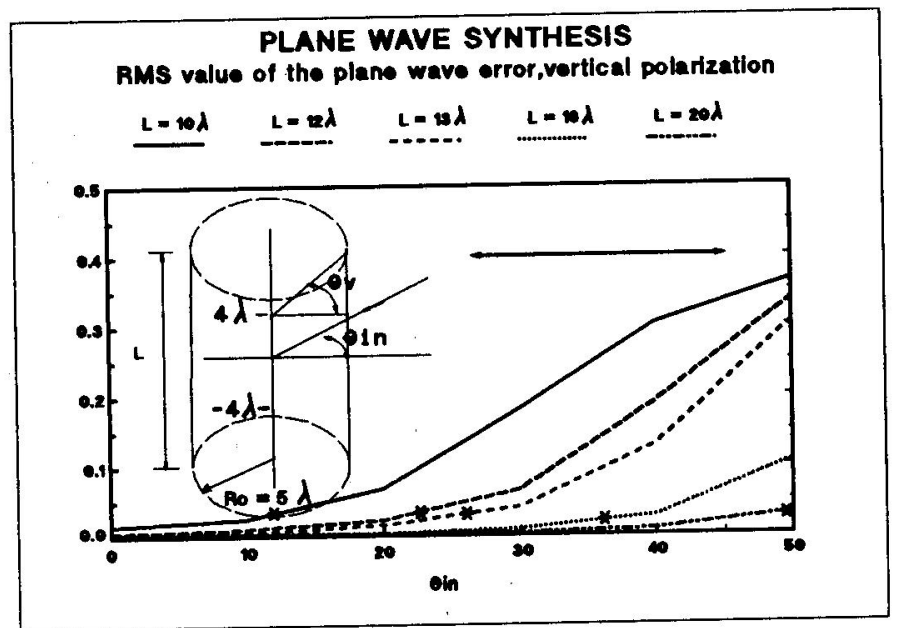

Figure 4. Rms value of the plane wave error. 